\title{
Détermination des tailles de particules métalliques et de leur distribution en catalyse hétérogène (*)
}

\author{
G. A. Martin \\ Institut de Recherches sur la Catalyse, 2, avenue Albert-Einstein, 69626 Villeurbanne Cedex, France
}

(Reçu le 21 novembre 1980, révisé le 26 janvier 1981, accepté le 4 février 1981)

\begin{abstract}
Résumé. - La mesure de la taille des grains de catalyseurs est indispensable au calcul de l'activité par unité d'aire active et aux études de frittage. Elle s'effectue en combinant plusieurs techniques : chimisorption ou physisorption des gaz, microscopie électronique, diffusion centrale des rayons $\mathrm{X}$, élargissement des raies de diffraction et magnétisme. Quelques résultats obtenus par la mise en œuvre simultanée de ces techniques sur des poudres métalliques ( $\mathrm{Pt}, \mathrm{Ni})$ supportées ou non, permettent d'apprécier les limites et les performances de ces méthodes granulométriques.
\end{abstract}

\begin{abstract}
In heterogeneous catalysis particle size measurements are required both to calculate catalytic activity per unit area of active phase and to study catalysts sintering. They can be performed by combining various techniques : chemisorption, physical adsorption of gases, electron microscopy, X-ray small angle scattering, X-ray line broadening and magnetism. In this paper, a brief description of these methods is presented. The limits of each technique is underlined by examining some examples (supported and unsupported nickel and platinum catalysts).
\end{abstract}

1. Introduction. - La vitesse, $r$, d'une réaction catalytique est généralement exprimée de la manière suivante :

$$
r=\frac{1}{Q} \frac{\mathrm{d} X}{\mathrm{~d} t},
$$

où $t$ est le temps (en secondes), $X$ (en moles) le degré d'avancement de la réaction et $Q$ la masse, le volume ou l'aire du solide catalytique. La mesure de la vitesse sert à caractériser l'activité du catalyseur.

Comme la catalyse est un phénomène de surface, il est a priori plus intéressant de calculer l'activité catalytique en la ramenant à l'unité d'aire du solide (activité airique ou intrinsèque), plutôt qu'à l'unité de masse (activité spécifique). La comparaison des activités intrinsèques permet de classer les réactions en deux types [1] :

- les réactions faciles, ou insensibles à la structure, pour lesquelles l'activité intrinsèque ne dépend pas de l'état de division du solide, du diamètre des particules, de l'orientation des plans de surface (ex. : échange $\mathrm{H}_{2}-\mathrm{D}_{2}$ );

- les réactions sensibles à la structure dans le cas contraire (ex. : $\mathrm{C}_{2} \mathrm{H}_{6}+\mathrm{H}_{2} \rightarrow 2 \mathrm{CH}_{4}$ ).

(*) Conférence présentée à la Réunion 1979 du Groupe Français de Spectrométrie Mössbauer.
Les recherches expérimentales sur la sensibilité à la structure des réactions catalytiques et les interprétations proposées font l'objet d'un nombre croissant de travaux, incluant les études sur poudres, films et monocristaux.

Par ailleurs, les mesures de taille moyenne et de dispersion des grains sont indispensables dans les études de frittage des catalyseurs, phénomène qui pourrait contribuer de manière notable au vieillissement.

Ceci explique l'importance prise en catalyse par la détermination de l'aire spécifique du catalyseur et de la dimension des petits grains qui constituent le solide. Ces mesures se font généralement :

- soit par adsorption de gaz (adsorption physique ou chimique),

- soit à l'aide de méthodes physiques, comme la microscopie électronique, les rayons $\mathrm{X}$, et le magnétisme. Les résultats les plus fiables sont évidemment obtenus par l'utilisation simultanée de plusieurs techniques.

Dans la première partie, nous décrirons sommairement les principes et les techniques permettant d'accéder à l'état de dispersion des solides catalytiques, et dans la seconde partie nous examinerons quelques résultats obtenus par la mise en œuvre de ces techniques et nous en comparerons les perfor- 
mances sur quelques exemples significatifs (catalyseurs métalliques).

2. Principes et techniques d'étude de la dispersion des catalyseurs. - L'étude de la dispersion (aire spécifique, dimension des petits grains) est effectuée à l'aide de mesures de quantités de gaz adsorbé et de méthodes physiques.

2.1 AIRE SPÉCIFIQUE PAR ADSORPTION PHYSIQUE. L'adsorption est le phénomène par lequel des molécules d'une phase fluide sont fixées par la surface d'un solide à son contact. Les forces mises en jeu peuvent être d'origine chimique (chimisorption) ou de même nature que les forces intermoléculaires dans les liquides (forces de Van der Waals). Dans ce dernier cas (physisorption), le problème consiste à repérer sur l'isotherme d'adsorption (courbe reliant le volume de gaz adsorbé à la pression d'équilibre) le point singulier correspondant à la formation de la monocouche. Diverses méthodes ont été proposées pour la détermination de ce point singulier. Brunauer, Emmet et Teller [2] ont admis que dès le début de l'adsorption des couches polymoléculaires sont présentes à la surface. Ils ont mis au point une méthode de calcul de l'aire basée sur cette hypothèse. Selon ces auteurs, l'adsorption multimoléculaire est régie par :

$$
\frac{P}{V\left(P_{0}-P\right)}=\frac{1}{V_{\mathrm{m}} C}+\frac{C-1}{V_{\mathrm{m}} C} \frac{P}{P_{0}}
$$

$V \quad$ volume adsorbé à pression $P$,

$V_{\mathrm{m}}$ volume de gaz correspondant à la monocouche, $C$ constante (fonction de la chaleur d'adsorption),

$P_{0}$ pression de vapeur saturante du gaz dans les conditions de l'adsorption.

En portant $P / P_{0}$ en fonction de $P / V\left(P_{0}-P\right)$, on observe une droite (pour $P / P_{0}$ compris entre 0,05 et 0,35$)$ dont la pente est $\beta=1 / V_{\mathrm{m}}$ lorsque $C$ est grand devant 1 (cas de $\mathrm{N}_{2}$ ). Pour calculer l'aire de la poudre il faut faire une hypothèse sur la surface occupée par une molécule adsorbée. Il est généralement admis que celle-ci est la même que celle qu'elle aurait à l'état liquide. Dans le cas de l'adsorption d'azote, on a la relation numérique suivante :

$$
S\left(\mathrm{~m}^{2}\right)=4,35 V_{\mathrm{m}}(\mathrm{ml} \mathrm{TPN}) \text {. }
$$

La détermination de l'aire spécifique des poudres par cette méthode (méthode B.E.T.) est universellement répandue. $\mathrm{Si}$ on admet que la poudre est constituée de sphères, leur diamètre $D$ est donné par :

$$
D(\mathrm{~nm})=\frac{0,6 \times 10^{4}}{\rho S\left(\mathrm{~m}^{2} / \mathrm{g}\right)},
$$

$\rho$, masse spécifique en $\mathrm{g} / \mathrm{cm}^{3}$.
2.2 AIRE SPÉCIFIQUE PAR CHIMISORPTION. - La plupart des masses de contact usuelles sont constituées d'une phase active, dispersée sur un support que nous supposerons inerte qui permet de maintenir la phase active dans un état de dispersion élevé. La méthode précédente qui fait appel à une physisorption, peu spécifique de la nature du substrat solide, permet de mesurer la surface totale. Or il importe de pouvoir accéder à l'aire de la surface active. Pour cela, il est possible de faire appel à la chimisorption de gaz simples tels que $\mathrm{H}_{2}, \mathrm{O}_{2}, \mathrm{CO} \ldots$ Ces gaz présentent l'avantage de ne pas se physisorber dans les conditions habituelles où ils sont utilisés.

La quantité fixée à saturation permet de se faire une idée de l'aire moyennant certaines hypothèses sur la stœchiométrie de surface et la répartition des plans cristallographiques à la surface. Prenons l'exemple de la chimisorption de $\mathrm{H}_{2}$ sur un métal de structure cubique à faces centrées $(\mathrm{Ni})$; nous admettrons :

- une équirépartition des plans de faible indice à la surface, (111), (110), et (100),

- que $\mathrm{H}_{2}$ se dissocie,

- qu'à chaque $\mathrm{H}$ correspond un atome métallique de surface (stœchiométrie de surface égale à l'unité).

Pour $\mathrm{Ni}$ on a alors la relation :

$$
S\left(\mathrm{~m}^{2}\right)=3,64 V_{\mathrm{s}}(\mathrm{ml} \text { TPN })
$$

$V_{\mathrm{s}}=$ volume chimisorbé à saturation.

Avant chimisorption, l'échantillon doit subir un dégazage thermique, ce qui constitue un inconvénient majeur pour les solides se frittant facilement. La méthode de titrage d'une espèce chimisorbée par un gaz réactif (par exemple, réaction de $\mathrm{O}_{2}$ chimisorbé par $\mathrm{H}_{2}$ [3] ou l'inverse), dispense d'un tel traitement; c'est là l'un de ses nombreux avantages. Nous en verrons le détail plus loin, dans son application à la détermination de l'aire du platine.

A côté de ces méthodes statiques, diverses méthodes dynamiques ont été proposées [4]. Elles impliquent diverses étapes :

- réduction de l'échantillon dans un courant d'hydrogène,

- mise en équilibre de l'échantillon à une température relativement faible dans un courant d'un gaz inerte (argon, hélium...) pouvant contenir des traces d'hydrogène,

- chauffage de l'échantillon dans le même courant gazeux pour éliminer $\mathrm{H}_{2}$ adsorbé qui est mesuré dans un détecteur catharométrique.

Une variante consiste à injecter ensuite dans le courant porteur des quantités connues d'un gaz chimisorbable jusqu'à saturation de l'échantillon. La quantité chimisorbée se calcule en tenant compte des quantités de gaz n'ayant pas réagi (quantités que l'on peut déterminer dans la cellule catharométrique). Ces méthodes dynamiques qui permettent de mesurer les 
quantités adsorbées ou désorbées peuvent également être utilisées pour les titrations. Elles sont rapides et ne nécessitent pas d'appareillages de vide ni de dispositifs gravimétriques ou volumétriques. Elles connaissent de ce fait un succès croissant; toutefois il est indispensable de les étalonner à l'aide des méthodes plus classiques.

Les déterminations d'aire métallique par chimisorption, telles que nous venons de les décrire, présentent toutefois certaines difficultés :

- la définition de la quantité chimisorbée à saturation est ambiguë :

- une quantité non négligeable de gaz peut se fixer sur le support en particulier lorsque la teneur métallique est très faible et la surface du support élevée (catalyseur de réformage); des mesures effectuées sur le support sans métal permettent toutefois de tenir compte de cette correction;

- $\mathrm{H}_{2}$ peut se dissoudre dans le métal (cas de Pd). Cet inconvénient peut être surmonté en opérant à une température et une pression aussi basse que possible, ou en chimisorbant $\mathrm{D}_{2}$, moins soluble;

- $\mathrm{O}_{2}$ peut réagir sur plusieurs couches métalliques;

- CO se lie avec le métal en donnant un mélange d'espèces linéaires (M.CO) pontées $\left(\mathrm{M}_{2} \mathrm{CO}\right)$, multiples $\left(\mathrm{M}_{x} \mathrm{CO}, x>3\right)$ ou précarbonyles $\left(\mathrm{MCO}_{y}\right)$ d'où une indétermination dans la stoechiométrie de surface, qui peut être levée dans certains cas par une étude spectroscopique infrarouge.

En conclusion, nous voyons que quelle que soit la méthode utilisée (statique ou dynamique) chaque type de gaz présente une difficulté propre pour déterminer l'aire d'un cataiyseur; c'est pourquoi dans la plupart des cas, il nous semble indispensable de faire appel à la chimisorption de plusieurs gaz et de comparer les résultats obtenus par chacun d'eux, et (ou) de mettre en œuvre une ou plusieurs techniques physiques.

2.3 MicrosCopie ÉleCtronique. - La microscopie est une technique particulièrement attirante, car elle permet de voir les particules métalliques $[5,6]$. Le pouvoir séparateur des microscopes actuellement commercialisés atteint $0,3 \mathrm{~nm}(1 \mathrm{~nm}=10 \AA)$ en résolution ponctuelle, et $0,1-5-0,2 \mathrm{~nm}$ sur réseau lorsque la tension accélératrice des électrons est de $100 \mathrm{kV}$.

Le contraste de l'image est dû à la fois à un phénomène de diffusion et de diffraction des électrons pour les particules relativement grandes, et à un phénomène de contraste de phase pour les particules métalliques très petittes. Ce contraste dépend à la fois des différences d'épaisseur de la préparation et du numéro atomique de l'élément. Il est ainsi relativement facile d'observer des grains de métaux ayant des poids atomiques élevés ( $\mathrm{Pt}, \mathrm{Pd})$ sur des supports composés de métaux légers $\left(\mathrm{Al}_{2} \mathrm{O}_{3}, \mathrm{SiO}_{2}, \mathrm{MgO}\right)$.

L'épaisseur de la préparation ne doit pas dépasser quelques dizaines de nanomètres d'où la nécessité de préparations appropriées, telles que coupes, répliques, ... Après examen d'un nombre élevé de préparation d'un même échantillon, il est possible d'obtenir la distribution de la taille des particules, et, connaissant leur forme, d'en déduire l'aire spécifique du solide.

Le microscope peut également être utilisé en microdiffracteur : le diagramme de diffraction obtenu sur l'écran correspond à une plage sélectionnée sur l'image de $2,5 \mathrm{~nm}^{2}$ environ, ce qui permet de définir la nature et éventuellement l'orientation du cristal observé par rapport au faisceau électronique. Le microscope peut également être utilisé en diffracteur simple, dans ce cas la plage étudiée correspond à l'ensemble de la préparation.

L'adaptation d'une platine goniométrique (résolution de $0,5 \mathrm{~nm}$ par exemple) permet des études de structure plus fines.

Une autre possibilité consiste à faire l'image de l'objet à l'aide d'un des faisceaux diffractés du métal. On obtient ainsi l'image des cristaux orientés dans une même direction. Cette technique dite du champ sombre, permet d'atténuer l'image du support, d'observer les cristallites des poudres non supportées telles que le noir de platine, et de mettre en évidence les défauts existant dans les petits cristaux.

La microscopie peut donc apporter beaucoup d'informations, mais le temps nécessaire à l'examen (préparation de l'échantillon, examen au microscope, dépouillement des clichés) est relativement long car il s'agit d'une méthode statistique : il est nécessaire d'examiner de nombreuses préparations pour avoir un ensemble de résultats représentatifs de l'échantillon, étant donné l'infime quantité de matière examinée à chaque fois. Les tailles limites de particules métalliques observables dépendent du pouvoir séparateur.

2.4 RaYons X. - Lorsque l'on effectue une analyse radiocristallographique par diffraction $X$ pour identifier la nature des phases présentes dans un catalyseur métallique, on constate :

1) un élargissement des raies de diffraction,

2) l'apparition d'un pic central de diffusion proche du faisceau.

Ces deux phénomènes sont à la base de deux méthodes qui permettent d'atteindre la taille des grains [7].

2.4.1 Elargissement des raies de diffraction des rayons X. - L'élargissement des raies de diffraction a plusieurs origines. L'une des principales est le faible diamètre des cristallites. Nous entendons par cristallite tout bloc cristallin qui donne naissance à un phénomène de diffraction cohérente, c'est-à-dire, où la périodicité tridimensionnelle de l'assemblage d'atomes est parfaite. Scherrer [8] a formulé la relation qui existe entre le diamètre des cristallites $D$, 
et la largeur $\beta$ d'une raie de diffraction de la manière suivante :

$$
D=\frac{\lambda}{\beta \cos \theta_{0}}
$$

$\lambda$ longueur d'onde,

$\theta_{0} \quad$ abscisse angulaire du maximum de la raie.

Si on utilise la largeur intégrale, largeur du rectangle ayant même surface et même hauteur que la raie, le diamètre calculé est un diamètre moyen en volume $D_{\mathrm{v}}$ (la définition exacte des diamètres moyens est donnée plus loin).

En fait, comme pour tout phénomène de type spectroscopique, l'appareillage introduit une distorsion se manifestant par un élargissement supplémentaire $b$. L'élargissement observé, $B$, doit être corrigé de cet élargissement instrumental. Cet élargissement instrumental peut être atteint expérimentalement par diffraction de l'échantillon massif bien cristallisé correspondant. Selon le but recherché, cette correction peut se faire de deux manières différentes :

a) à l'aide de formules simples dérivées d'hypothèses concernant la forme analytique des profils. On aboutit alors, selon l'hypothèse à des formules du type suivant $[8,9]$ :

$$
\begin{aligned}
& \beta=\left(B^{2}-b^{2}\right)^{1 / 2} \quad \text { (formule de Warren) } \\
& \beta=\frac{B^{2}-b^{2}}{B} \quad \text { (formule d'Anantharaman) } \\
& \beta=B-b \quad \text { (formule de Scherrer) }
\end{aligned}
$$

b) à l'aide de procédés plus élaborés utilisant la déconvolution ou la transformée de Fourier, on peut obtenir le diamètre moyen sans faire d'hypothèse sur la forme des profils. Si on compare les résultats obtenus par les méthodes de correction simples à ceux donnés par les méthodes élaborées, on constate que c'est la formule d'Anantharaman qui donne la meilleure approximation dans de très nombreux cas. Avec ces procédés, on peut également obtenir, outre le diamètre moyen, la courbe de répartition des diamètres des cristallites.

Les diamètres moyens et les courbes de répartition ainsi calculées correspondent à des dimensions perpendiculaires à un plan $h \mathrm{kl}$ déterminé. L'étude de l'élargissement de chacune des raies de diffraction (perpendiculairement aux plans (111), (200), (220), par exemple dans le cas de $\mathrm{Ni}$ ) permet de se faire une idée assez précise de la forme du cristallite.

L'avantage de la méthode de mesure de la dimension par élargissement des raies de diffraction est qu'elle permet d'accéder au diamètre moyen d'une manière simple, rapide, et peu onéreuse; c'est la raison pour laquelle de très nombreux laboratoires de catalyse l'utilisent. Les dimensions de cristallites métalliques qui peuvent être ainsi mesurées sont comprises entre 40 et $2 \mathrm{~nm}$ environ. Toutefois, dans le cas des métaux supportés, les raies de diffraction du support risquent d'interférer avec celles du métal. De plus, la charge métallique doit dépasser $3 \%$ pour $\mathrm{Pt}, 4 \%$ pour $\mathrm{Pd}$ et $6 \%$ pour $\mathrm{Ni}$ (cette limite est d'autant plus faible que le facteur de diffusion est plus grand).

Enfin nous devons attirer l'attention sur la nature exacte de l'information que l'on peut tirer de l'élargissement des raies de diffraction : cette technique permet d'atteindre la dimension du cristallite et non celle du grain. Un grain peut être en effet constitué de plusieurs cristallites, cette méthode permet alors de détailler la texture interne du grain. L'élargissement des raies de diffraction permet de calculer l'aire métallique lorsque les grains sont monocristallins, et uniquement dans ce cas (les petits grains de Pt semblent être monocristallins la plupart du temps).

2.4.2 Diffusion centrale des rayons $X$. - La relation de Bragg

$$
\lambda=2 d \sin \theta
$$

montre que l'angle de diffraction $\theta$ varie comme l'inverse de la distance $d$ séparant deux plans cristallins du réseau. Dans la plupart des substances cristallines, cette distance est du même ordre de grandeur que la longueur d'onde de sorte que $\theta$ est relativement grand. Dans certaines molécules complexes (polymères, protéines...) la distance entre plans cristallins atteint quelques nanomètres, de sorte qu'il a été nécessaire d'étudier les intensités diffractées à des angles très faibles ( $2^{\prime}$ pour $100 \mathrm{~nm}$ avec $\left.\mathrm{CuK}_{\alpha}\right)$. De l'étude des grandes équidistances dans les cristaux parfaits, on est passé à l'étude d'imperfections dans les cristaux donnant également lieu à une diffusion continue dans un domaine correspondant à des équidistances de 1 à $100 \mathrm{~nm}$. La diffusion centrale est l'étude de la correspondance entre cette courbe aux petits angles et la distribution des défauts tels que pores, micro-particules, agrégats dans les alliages, variation de cristallinité dans les polymères, etc...

D'une manière générale, toute variation continue ou discontinue de la densité électronique (et donc de la densité) produit une diffusion que l'on relie par transformation de Fourier à la dimension et à la forme des défauts produisant la variation de densité.

Pour les métaux purs ou supportés, on peut déterminer la surface métallique en appliquant la théorie de Porod [10] qui ne repose sur aucune hypothèse de forme. On peut également déterminer la distribution de la longueur des cordes (cordes : segments ayant toutes les orientations possibles et dont les extrémités s'appuient sur les contours de la particule), ou enfin si on fait l'hypothèse de particules sphériques, on obtient la distribution des diamètres.

Les diamètres limites qui peuvent être mesurés sont 500 et $1 \mathrm{~nm}$ (les angles correspondants sont de quelques minutes à quelques degrés). En fait ces limites sont d'origine technologique et il n'existe aucun obstacle théorique qui s'oppose à la mesure de dimension d'agrégats de quelques atomes bien que dans ce 
cas il ne s'agisse pas à proprement parlé de diffusion centrale; les limitations expérimentales sont les suivantes : luminosité suffisante de la source, et dispositif permettant d'enregistrer en un temps raisonnable un phénomène très étalé dans l'espace.

Lorsque la phase active est déposée sur un support (problème à trois phases), les deux phases solides vont ajouter leurs effets, et pour obtenir la diffusion proprement métallique, on retranche l'intensité diffusée par le catalyseur de celle diffusée par le support avant échange ou imprégnation. Les intensités diffusées varient comme le carré de la densité de la matière. Ainsi, la limite en concentration métallique sera d'autant plus faible que l'on passe du nickel au platine

$$
\mathrm{Ni}<\mathrm{Pd}<\mathrm{Pt}<,
$$

et de, $\mathrm{ThO}_{2}$ à $\mathrm{SiO}_{2}$ comme support :

$$
\mathrm{ThO}_{2}<\mathrm{Al}_{2} \mathrm{O}_{3}=\mathrm{MgO}<\mathrm{SiO}_{2} \text {. }
$$

Dans la pratique, les concentrations métalliques limites sont de l'ordre du pourcent. Il n'existe pas de limitations théoriques, il suffit que la différence entre les intensités diffusées par les deux systèmes ne soit pas entachée d'une trop grande erreur statistique. Cette méthode suppose que le processus de dépôt du métal ne modifie pas la morphologie propre du support. La validité de cette hypothèse peut dans certains cas être mise en doute. C'est pourquoi il est apparu une nouvelle technique, pour masquer les effets propres dus au support, et qui consiste à imprégner le catalyseur de liquides possédant une densité élevée (iodure de méthyle...) et proche de celle du support. On peut ainsi faire disparaitre complètement la diffusion par le support [11].

2.5 MÉTHOdeS MAGNÉTIQUES. - Un certain nombre de catalyseurs industriels sont ferromagnétiques ( $\mathrm{Fe}, \mathrm{Co}, \mathrm{Ni}$, alliages). Leur état de dispersion leur confère des propriétés remarquables que l'on a mis à profit pour en estimer le degré de dispersion.

Les ferromagnétiques massifs sont constitués par la juxtaposition d'un grand nombre de domaines, les domaines de Weiss, à l'intérieur desquels les moments magnétiques des atomes sont parallèles. En dessous d'une certaine taille critique ( $30 \mathrm{~nm}$ pour $\mathrm{Ni}$ ), la forme la plus stable est celle du monodomaine : tous les moments atomiques à l'intérieur du grain sont orientés dans une même direction; le moment résultant est égal à $N \mu_{0}, N$ est le nombre d'atomes du grain et $\mu_{0}$ le moment d'un atome. Soumis au champ $H$, il subit le couple $N \mu_{0} H$ qui tend à l'orienter. L'orientation est contrecarrée par l'agitation thermique $k T$. L'aimantation $M$ (résultante des moments dans la direction de $H$ ) est régie par la loi de Langevin, valable si $N \mu_{0}$ est grand :

$$
\begin{aligned}
M / M_{\mathrm{s}} & =L\left(\frac{N \mu_{0} H}{k T}\right) \\
L(a) & =\operatorname{coth} a-1 / a .
\end{aligned}
$$

Les courbes $M(H)$ à température constante sont paramétrées par $N$, qui est une fonction du volume du grain. Leur détermination expérimentale permet d'accéder au diamètre de la sphère de même volume. $\mathrm{Si}$ on représente la distribution de tailles des grains à l'aide d'une fonction analytique simple, il est possible de déterminer celle-ci d'une manière précise par optimisation [12] (méthode de Langevin, ou $M(H)$ ).

La relation précédente n'est valable que pour des monodomaines en équilibre thermodynamique (superparamagnétisme). Lorsque les grains sont relativement gros, ou la température basse, ou encore le temps d'observation court, les moments restent bloqués dans leur direction de facile aimantation, provoquant une aimantation rémanente proportionnelle au volume total des grains correspondants. A une température $T$ et un temps d'observation $t$ donnés (100 $\mathrm{s}$ dans le cas des mesures magnétiques statiques) il est possible de faire correspondre un diamètre critique de grain $D_{\mathrm{c}}$ (volume critique correspondant : $\left.V_{\mathrm{c}}\right)$ à l'aide de la relation suivante (Néel [13]) :

$$
1 / t=f_{0} \exp -\frac{V_{\mathrm{c}} I_{\mathrm{s}} H_{\mathrm{c}}}{2 k T}
$$

$f_{0}$ facteur de fréquence, voisin de $10^{9} \mathrm{~s}^{-1}$ pour $\mathrm{Ni}$, $I_{\mathrm{s}}$ aimantation spontanée volumique (500 uem cgs pour $\mathrm{Ni}$ ),

$H_{\mathrm{c}}$ champ coercitif à $0 \mathrm{~K}$.

A partir des variations de la rémanente avec la température (pour un temps d'observation donné), il est possible de calculer la distribution complète de la taille des grains, c'est la méthode de Weil [14].

Il existe une autre méthode basée sur ce modèle qui consiste à opérer à température constante, à modifier le temps d'observation en appliquant un champ alternatif de fréquence variable, et qui nous a permis d'obtenir des résultats intéressants [15] (méthode de la fréquence).

La méthode de Weil nécessite des températures basses (hélium liquide) et ne peut être guère mise en œuvre que dans les grands laboratoires spécialement équipés. Par contre, la méthode de détermination de la taille des grains par mesure de la courbe $M(H)$ est beaucoup plus rapide ( $1 \mathrm{~h}$ environ) et ne nécessite pas de bains cryogéniques dans la plupart des cas. Elle se prête particulièrement bien à des études systématiques. Les diamètres métalliques ne doivent pas être supérieurs à $300 \mathrm{~nm}$; il n'existe pas de limites inférieures (la précision sur $D$ est d'autant plus grande que le grain est plus petit). La grande dilution de la phase métallique sur un support ne constitue pas non plus un obstacle, car le ferromagnétisme est un phénomène coopératif très intense, facilement détectable.

\subsection{DÉFINITIONS ET NOTATIONS EN GRANULOMÉ-} TRIE. - Lorsque l'on est amené à comparer les résultats obtenus par chimisorption et l'une des techniques physiques que nous avons décrite, ou les résultats des techniques physiques entre eux, le pro- 
blème de la définition et de la notation se pose d'une manière critique.

La notion la plus utilisée pour un ensemble de particules sphériques est celle de loi granulométrique, ou loi des fréquences, que nous désignerons par $f_{N}(D)$. $f_{N}(D) \mathrm{d} D$ désigne la fraction du nombre total $N_{0}$ des particules dont le diamètre est compris entre $D$ et $\mathrm{d} D$; il vient immédiatement :

$$
\int_{0}^{\infty} f_{N}(D) \mathrm{d} D=1
$$

De la même manière, on définit une distribution des diamètres en surface $f_{\mathrm{s}}(D)$ tel que $f_{\mathrm{s}}(D) \mathrm{d} D$ représente la fraction de la surface totale $\left(S_{0}\right)$ des particules dont le diamètre est compris entre $D$ et $D+\mathrm{d} D$. Enfin, la distribution en volume $f_{\mathrm{v}}(D)$ se rapportera au volume des particules : $f_{\mathrm{v}}(D) \mathrm{d} D$ est la fraction de volume total $\left(V_{0}\right)$ des particules dont le diamètre est compris entre $D$ et $D+\mathrm{d} D$. Par définition, nous aurons de la même manière :

$$
\int_{0}^{\infty} f_{\mathrm{s}}(D) \mathrm{d} D=1, \quad \int_{0}^{\infty} f_{\mathrm{v}}(D) \mathrm{d} D=1 ;
$$

il existe des relations simples entre $f_{N}, t_{\mathrm{s}}$ et $f_{\mathrm{v}}$ : exemple :

$$
f_{\mathrm{v}}=\frac{f_{n} D^{3}}{\int_{0}^{\infty} f_{n} D^{3} \mathrm{~d} D}
$$

Nous pourrons définir des diamètres moyens de nombre, surface et volume de la façon suivante :

$$
\begin{aligned}
& \bar{D}_{n}=\int_{0}^{\infty} D f_{n}(D) \mathrm{d} D \\
& \bar{D}_{\mathrm{s}}=\int_{0}^{\infty} D f_{\mathrm{s}}(D) \mathrm{d} D \\
& \bar{D}_{\mathrm{v}}=\int_{0}^{\infty} D f_{\mathrm{v}}(D) \mathrm{d} D .
\end{aligned}
$$

Le diamètre moyen de surface présente un intérêt particulier car il est relié à la surface spécifique $S$ de l'ensemble des grains sphériques par la relation :

$$
S=\frac{0,6 \times 10^{4}}{\rho D_{\mathrm{s}}}
$$

$S$ en $\mathrm{m}^{2} / \mathrm{g}, \rho$ masse spécifique en $\mathrm{g} / \mathrm{cm}^{3}$ et $D_{\mathrm{s}}$ en $\mathrm{nm}$.

Il est intéressant de remarquer que ces définitions s'appliquent aux mesures de granulométrie effectuées sur des clichés de microscopie électronique dans le cas de particules sphériques; en effet, lorsqu'on dénombre $n$ particules dont le diamètre est compris entre $D$ et $D+\Delta D$, on constate que $n_{i}(D) / \sum n_{i}$ n'est autre que la loi granulométrique définie ci-dessus, donc :

$$
\bar{D}_{n}=\frac{\sum_{i} n_{i} D_{i}^{2}}{\sum_{i} n_{i} D_{i}} \bar{D}_{\mathrm{s}}=\frac{\sum_{i} n_{i} D_{i}^{3}}{\sum_{i} n_{i} D_{i}^{2}} \quad \bar{D}_{\mathrm{v}}=\frac{\sum_{i} n_{i} D_{i}^{4}}{\sum_{i} n_{i} D_{i}^{3}} .
$$

Les techniques magnétiques peuvent faire intervenir des diamètres moyens plus complexes, et l'on simplifie l'écriture en introduisant les moments d'ordre $n, M_{n}$ définis de la façon suivante :

$$
M_{n}=\int D^{n} f_{v}(D) \mathrm{d} D .
$$

On voit immédiatement que $\bar{D}_{\mathrm{v}}=M_{1}$, et un calcul plus complexe montre que $D_{\mathrm{s}}=M_{-1}^{-1}$. La loi d'aimantation devient $M=M_{\mathrm{s}} \int f_{\mathrm{v}}(D) \frac{\rho H}{k T} \mathrm{~d} D$, et les diamètres moyens calculés d'après la partie relative aux faibles champs, et aux forts champs peuvent s'écrire :

$$
D_{1}=M_{3}^{1 / 3} \quad D_{2}=M_{-3}^{-1 / 3} .
$$

La relation

$$
M_{3}^{1 / 3} \leqslant M_{-1}^{-1} \leqslant M_{-3}^{-1 / 3}
$$

entraîne

$$
\bar{D}_{1} \leqslant \bar{D}_{\mathrm{s}} \leqslant \bar{D}_{2}
$$

et en première approximation nous écrirons :

$$
\bar{D}_{、}=\frac{\bar{D}_{1}+\bar{D}_{2}}{2} \text {. }
$$

3. Exemples de détermination et dimension de petits grains et comparaison des méthodes. - Les exemples sont choisis parmi les catalyseurs métalliques. Nous distinguerons le cas des métaux non supportés des métaux sur support.

3.1 MÉTAUX NON SUPPORTÉS EN POUDRE. - Ils sont obtenus par diverses techniques : décomposition sous vide de sels organiques ( $\mathrm{Ni}$ et $\mathrm{Cu}$ ex formiates ou oxalates), réduction d'oxydes ou de sels métalliques par $\mathrm{H}_{2}$ (noirs de platine, nickel de Sabattier), décomposition de métaux carbonyles ( $\mathrm{Ni}$ Mond), attaque sélective par $\mathrm{Na}(\mathrm{OH})$ d'alliages $\mathrm{M}-\mathrm{Al}$ avec $\mathrm{M}=\mathrm{Ni}, \mathrm{Fe}, \mathrm{Co}, \mathrm{Cu}, \mathrm{Ag}, \mathrm{Pt}, \mathrm{Pd}$, etc... (métaux de Raney ou métaux squelettes). Leur aire métallique est directement accessible par adsorption physique de gaz (BET), et leur détermination ne présente pas de difficultés particulières. Il est toutefois intéressant de comparer les résultats ainsi obtenus à ceux tirés des méthodes physiques et de la chimisorption, de manière à en apprécier les capacités, et à en extrapoler les caractéristiques aux métaux supportés pour lesquels la physisorption donne l'aire totale, et non l'aire proprement métallique. Nous considérons les quelques exemples suivants : 
3.1.1 Noirs de platine. - Les gaz les plus souvent utilisés pour mesurer la surface des noirs de platine sont $\mathrm{H}_{2}$ et $\mathrm{O}_{2}$. Si $\mathrm{Pt}_{\mathrm{s}}$ désigne un atome de surface, il importe de connaitre les stœechiométries de surface, $x, y$.

$$
\begin{aligned}
& \mathrm{Pt}_{\mathrm{s}}+\frac{x}{2} \mathrm{O}_{2} \rightarrow \mathrm{Pt}_{\mathrm{s}}-\mathrm{O}_{x} \\
& \mathrm{Pt}_{\mathrm{s}}+\frac{y}{2} \mathrm{H}_{2} \rightarrow \mathrm{Pt}_{\mathrm{s}}-\mathrm{H}_{y} .
\end{aligned}
$$

En comparant les volumes adsorbés à $20^{\circ} \mathrm{C}$ sous 50 torr aux surfaces BET (quelques $\mathrm{m}^{2}$ ), et en admettant une équirépartition des 3 plans de bas indices à la surface du métal, Boudart et coll. [16] trouvent :

$$
x=0,84 \mp 0,01 \text { et } y=0,86 \mp 0,02,
$$

soit approximativement un atome $(\mathrm{H}, \mathrm{O})$ par platine de surface. En ce qui concerne $\mathrm{CO}$ [21], le rapport est voisin de 0,75 $\left(\mathrm{Pt}_{\mathrm{s}}-\mathrm{CO} 0,75\right)$. Sur platine de Raney, Candy et coll. [17] ont observé des stœchiométries de surface assez différentes : $x=0,8$ et $y=1,9$. Les écarts observés proviennent vraisemblablement des différences de protocole d'adsorption.

3.1.2 Ni de Raney. - Nous avons représenté sur la figure 1 la quantité d'hydrogène que l'on peut récupérer par traitement thermique sous vide d'échantillons de diverses surfaces spécifiques [19,20], ainsi que la droite calculée en admettant la stœechiométrie de

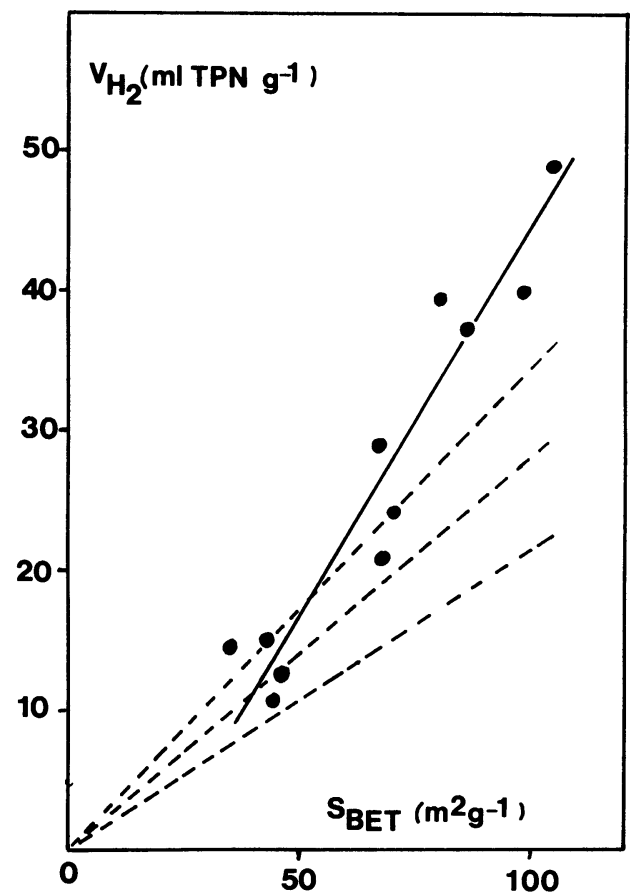

Fig. 1. - Volume d'hydrogène désorbé en fonction de la surface BET de diverses poudres de nickel de Raney. En pointillé, la droite calculée en admettant la stæchiométrie de surface $\mathrm{Ni} / \mathrm{H}=1$ et la présence de plans (111), (110) et (100) en surface.

[Volume of desorbed hydrogen as a function of the BET surface area for various Raney nickel powders. Dashed line : calculated straight line assuming that the surface stoechiometry $\mathrm{Ni}-\mathrm{H}$ is equal to unity and that the nickel surface is made up of (111) (110) and (100) planes.] surface $\mathrm{NiH}$ et l'équirépartition des plans (111) (110) et (100); les points expérimentaux relatifs aux échantillons de faibles surfaces sont approximativement placés sur cette droite, ce qui montre que l'hypothèse sur la stoechiométrie est vraisemblablement correcte. De tels accords sont également observés pour les poudres de nickel d'origines diverses [21, $22,23]$. Toutefois, les points de la figure 1 relatifs aux échantillons plus divisés s'écartent notablement de la droite, indiquant l'existence d'un hydrogène «excédentaire » dont la nature n'a pas encore été parfaitement élucidée.

Cet exemple montre un premier type de difficulté que l'on rencontre dans la détermination d'une aire métallique par chimisorption : la présence de plusieurs états de chimisorption du gaz.

3.2 MÉTAUX SUPPORTÉs. - La plupart des métauX non supportés ont une aire métallique faible, dépassant rarement $10 \mathrm{~m}^{2} / \mathrm{g}$, de plus ils se frittent facilement sous l'effet de la chaleur. Le pourcentage des atomes métalliques utiles pour la catalyse est de ce fait peu élevé. La recherche de surfaces plus développées et thermiquement plus stables a conduit à déposer le métal sur un support : $\mathrm{SiO}_{2}, \mathrm{Al}_{2} \mathrm{O}_{3}, \mathrm{SiO}_{3}-\mathrm{Al}_{2} \mathrm{O}_{3}$, $\mathrm{TiO}_{2}, \mathrm{MgO} \ldots$ La charge métallique peut être relativement élevée (quelques dizaines de pourcents pour $\mathrm{Ni}$ ) mais la recherche d'une dispersion maximale ainsi que certaines considérations économiques dans le cas des métaux précieux ont conduit à ramener cette charge au pourcent, et même à la fraction de pourcent $(0,35 \%$ pour les catalyseurs de réformage).

La mesure de l'aire BET qui conduit à l'aire métallique totale lorsqu'il n'y a pas de support, donne dans ce cas la somme de l'aire métallique et de l'aire du support. Dès lors, des procédés sélectifs comme la chimisorption ainsi que les méthodes physiques doivent être mis en œuvre pour mesurer la surface et étudier la structure atomique superficielle. Nous examinerons quelques résultats obtenus sur des catalyseurs de nickel et de platine, tout en soulignant les possibilités et limites des diverses méthodes pour chacun des catalyseurs.

3.2.1 Nickel sur support. - Dans la plupart des cas, ces échantillons sont préparés à partir de sels métalliques déposés sur un support inerte divisé tel que la silice, puis réduits à l'état métallique par un agent réducteur tel que l'hydrogène gazeux en général à température assez élevée.

Le degré de dispersion de la phase métallique peut être estimé par chimisorption de l'hydrogène qui, en principe, ne se fixe pas sur le support.

En comparant la quantité d'hydrogène adsorbée (ou désorbée) à l'aire BET, nous avions conclu que la stœechiométrie de surface $\mathrm{Ni}_{\mathrm{s}} / \mathrm{H}$ est égale à l'unité pour les échantillons non supportés. Yates, Taylor et Sinfelt [24] admettent la même stœchiométrie pour les échantillons supportés et proposent d'estimer l'aire métallique d'après la quantité fixée à $20^{\circ} \mathrm{C}$ sous 
10 torr. Sinfelt et ses collaborateurs ont proposé une variante, faisant appel au magnétisme [25] : la baisse relative d'aimantation à saturation due au recouvrement de la surface par $\mathrm{H}_{2}$, est égale au nombre relatif d'atomes métalliques de surface (cette propriété est une conséquence directe du fait bien établi que l'adsorption d'une molécule de $\mathrm{H}_{2}$ diminue l'aimantation à saturation d'une quantité égale à deux fois le moment magnétique de l'atome [26]. Toutefois, dans les conditions où opèrent ces auteurs, la saturation de la surface par l'hydrogène n'est pas certaine. Dans un récent travail [27], nous avons pu déterminer le volume de gaz adsorbé sur nickel à saturation : comme l'illustre la figure 2 le réseau d'isothermes d'adsorption en transformée de Freundlich $(\log P, \log V)$ converge en un point $\mathrm{S}$, correspondant à la saturation (chaleur

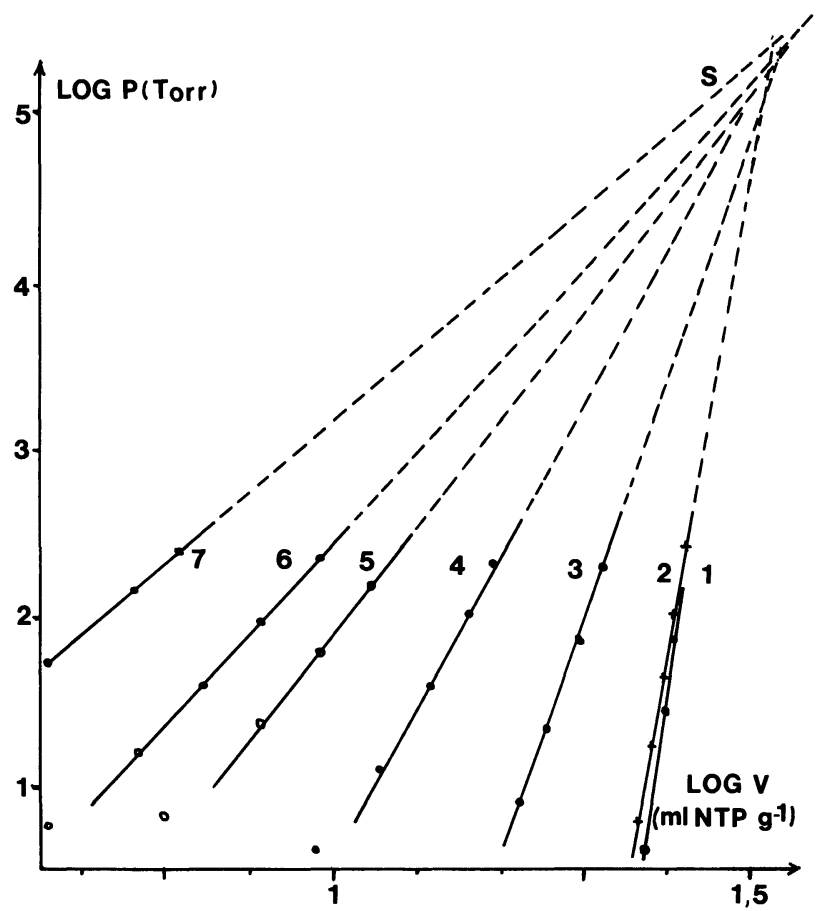

Fig. 2. - Isothermes d'adsorption d'hydrogène sur un catalyseur $\mathrm{Ni} / \mathrm{SiO}_{2}$ à $-78,-17,147,236,297,367$ et $520^{\circ} \mathrm{C}$ (courbes 1 à 7$)$.

[Adsorption isotherms of hydrogen on a $\mathrm{Ni} / \mathrm{SiO}_{2}$ catalyst at -78 , - $17,147,236,367$ and $520^{\circ} \mathrm{C}$ (curves 1 to 7 , respectively).]

d'adsorption nulle). En admettant que les particules sont approximativement sphériques, que les plans de surfaces sont les plans (100), (110) et (111) et que la stoechiométrie de surface est égale à l'unité $\left(\mathrm{Ni}_{\mathrm{s}} / \mathrm{H}=1\right)$, nous avons calculé les diamètres $D_{\mathrm{H}}$ rassemblés dans le tableau I. Nous pouvons constater qu'il y a excellent accord entre les diamètres ainsi obtenus et ceux calculés par méthodes magnétiques, $D_{\mathrm{s}}$ (d'après l'équation de Langevin), ce qui justifie a posteriori la validité des hypothèses avancées dans le cas des échantillons considérés.

La mesure de l'aire par chimisorption de $\mathrm{O}_{2}$ soulève de nombreuses difficultés. La stoechiométrie varie selon la taille des grains, le degré de réduction... Ainsi pour $\mathrm{Ni} / \mathrm{SiO}_{2}$, le nombre d'atomes d'oxygène
Tableau I. - Comparaison des diamètres moyens de surface de grains de nickel calculés par adsorption d'hydrogène, $D_{\mathrm{H}}$, et par méthodes magnétiques

[Comparizon of surface average diameters of nickel particles calculated from hydrogen adsorption data and magnetism.]

$\begin{array}{cccc}\begin{array}{c}\text { Echantillons } \\ \mathrm{Ni} / \mathrm{SiO}_{2}\end{array} & \begin{array}{c}(1 \mathrm{~nm}=10 \AA) . \\ l_{11_{2}}\end{array} & \begin{array}{c}D_{\mathrm{H}} \\ (\mathrm{ml} \mathrm{TPN} / \mathrm{g} \mathrm{Ni})\end{array} & \begin{array}{c}\mathrm{D}_{\mathrm{m}} \\ (\mathrm{nm})\end{array} \\ -1 & - & - & - \\ 1 & 86 & 2,5 & 2,5 \\ 2 & 35 & 6,3 & 6,4 \\ 3 & 11 & 20 & 22\end{array}$

chimisorbé à $20^{\circ} \mathrm{C}$ par atome de nickel superficiel peut varier de 0,5 à 2 . Nous aimerions citer quelques difficultés supplémentaires qui ont été rencontrées au laboratoire dans la détermination de l'aire métallique des catalyseurs à base de nickel.

1) $\mathrm{Ni} / \mathrm{SiO}_{2}$ partiellement réduits : la quantité d'hydrogène est une fois plus faible [28] que celle que l'on peut prévoir à partir des tailles de grain mesurées par une méthode physique, une partie seulement de la surface du métal est accessible aux gaz, l'autre partie étant vraisemblablement recouverte par la phase non réduite.

2) $\mathrm{Ni} / \mathrm{SiO}_{2}$ ex silicate basique de nickel réduit totalement; dans ce cas également la surface calculée par adsorption d'hydrogène est nettement plus petite que celle déduite de l'examen au microscope électronique : une partie importante des grains de métal est enfouie dans le support et est inaccessible aux gaz, comme le montrent les clichés de microscopie électronique.

3) Sur $\mathrm{Ni} / \mathrm{Al}_{2} \mathrm{O}_{3}, \mathrm{Ni} / \mathrm{SiO}_{2}-\mathrm{Al}_{2} \mathrm{O}_{3}, \mathrm{Ni} / \mathrm{MgO}, \mathrm{H}_{2}$ se fixe sous deux formes magnétiquement très différenciées [29] : l'une correspond à l'adsorption sur le métal, et l'autre semble liée à la présence simultanée du métal et de la phase non réduite; dans ce cas la définition de la surface métallique est délicate.

La comparaison des résultats obtenus par la mise en œuvre de 4 méthodes physiques différentes sur un même échantillon est particulièrement intéressante [30] comme l'illustrent les figures 3 et 4 : l'accord entre ces courbes peut être considéré comme satisfaisant dans la mesure où on ne tient pas compte des résultats obtenus par la technique d'élargissement des raies de diffraction, qui conduit à des dimensions systématiquement plus petites. Cet écart est vraisemblablement dû au caractère polycristallin des grains de métal, chaque grain étant constitué par un nombre assez élevé de cristallites dont les dimensions déterminent l'élargissement de la raie de diffraction.

3.2.2 Platine sur support. - La méthode de mesure des aires du platine par titrage $\mathrm{H}_{2}-\mathrm{O}_{2}$ proposée par Benson et Boudart [31] est très largement utilisée : la surface métallique initialement recouverte en 


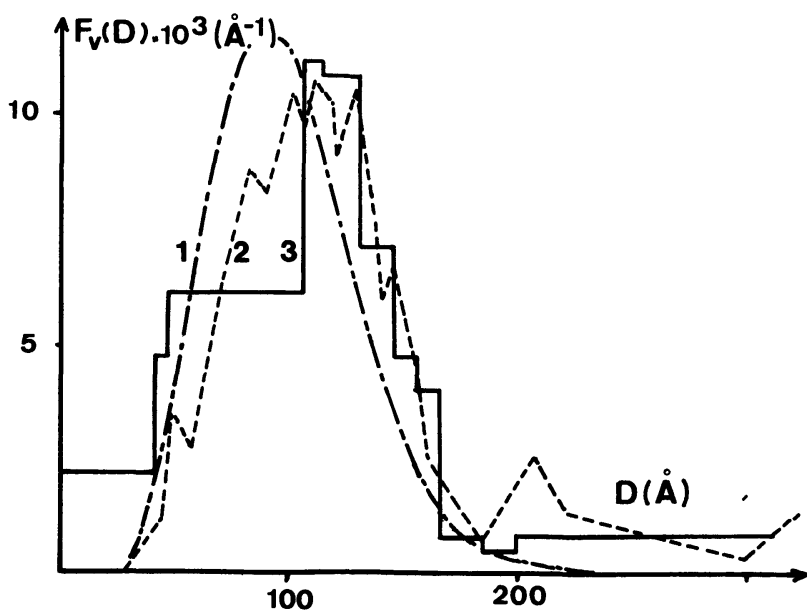

Fig. 3. Distributions de la taille des grains de nickel d'un échantillon $\mathrm{Ni} / \mathrm{SiO}_{2}$ calculées d'après : (1) les courbes aimantåtionchamp ; (2) diffusion centrale des rayons X ; (3) la méthode de Weil (rémanente).

[Distribution of nickel particles size of a $\mathrm{Ni} / \mathrm{SiO}_{2}$ sample calculated from : (1) curves magnetization vs. field strength; (2) X-Ray low angle scattering; (3) the Weil method (remanence).]

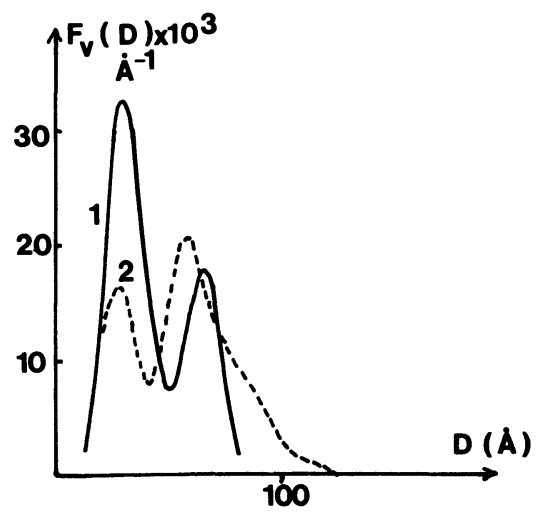

Fig. 4. - Distribution de. la taille des cristallites de nickel d'un échantillon $\mathrm{Ni} / \mathrm{SiO}_{2}$ analogue à celui de la figure 3 calculée par élargissement des raies de diffraction des rayons $X(10 \AA=1 \mathrm{~nm})$. Courbes 1 et 2, distribution des tailles perpendiculaires aux plans (200) et (111).

[Distribution of nickel particles size of a $\mathrm{Ni} / \mathrm{SiO}_{2}$ sample similar to that studied in figure 3 calculated from X-Ray line broadening. Curves 1 and 2, distribution of sizes perpendicular to (200) and (111) planes.]

Tableau III. - Diamètres des grains de $\mathrm{Pt}$ d'un catalyseur $\mathrm{Pt} / \mathrm{Al}_{2} \mathrm{O}_{3}$ calculés par diverses techniques $(1 \mathrm{~nm}=10 \AA)$.

[Platinum particle size of a $\mathrm{Pt}_{/} \mathrm{Al}_{2} \mathrm{O}_{3}$ catalyst obtained by various techniques $(1 \mathrm{~nm}=10 \AA)$.]

\begin{tabular}{cccc}
$\mathbf{P t}$ & $\begin{array}{c}\text { Echantillons } \\
\text { Traitement } \\
(\%)\end{array}$ & $\left({ }^{\circ} \mathrm{C}\right)$ & \multicolumn{2}{c}{ Adsorption } \\
- & - & $-\mathrm{H}_{2}$ & $\mathrm{CO}$ \\
0,6 & 500 & 1,2 & -1.3 \\
1 & 500 & 1,3 & 1,4 \\
2 & 500 & 1,6 & 1,8 \\
2,7 & 500 & 2,1 & 2,2 \\
3,7 & 500 & 2,7 & 2,9 \\
4,8 & 500 & 3,0 & 3,2 \\
3,7 & 600 & 3,1 & - \\
3,7 & 700 & 3,9 & - \\
3,7 & 800 & 3,9 & -
\end{tabular}

hydrogène adsorbé est mise en contact avec $\mathrm{O}_{2}$. L'hydrogène adsorbé est oxydé en eau qui se fixe sur le support $\left(\mathrm{Al}_{2} \mathrm{O}_{3}\right)$ et est remplacé par de l'oxygène adsorbé. Après pompage de l'oxygène moléculaire excédentaire, $\mathrm{H}_{2}$ est admis et il y a formation d'eau et adsorption de ce gaz à la surface. L'augmentation de poids du système est suivie par gravimétrie. Ces opérations peuvent être renouvelées un certain nombre de fois, augmentant d'autant la précision de la mesure. Les auteurs de cette méthode admettent que les stœchiométries $\mathrm{Pt}_{\mathrm{s}} / \mathrm{H}$ et $\mathrm{Pt}_{\mathrm{s}} / \mathrm{O}$ sont voisines de l'unité. Les résultats ainsi obtenus sont en général en bon accord avec ceux issus des techniques physiques comme la microscopie électronique, ainsi que l'illustrent les résultats du tableau II.

Tableau II. - Comparaison des diamètres de grains de platine d'un catalyseur $\mathrm{Pt} / \mathrm{Al}_{2} \mathrm{O}_{3} \gamma$, calculé par la méthode de titrage $\mathrm{H}_{2}+\mathrm{O}_{2}$ et microscopie électronique [32] $(1 \mathrm{~nm}=10 \AA)$.

[Comparizon of platinum particle diameters in a $\mathrm{Pt} / \mathrm{Al}_{2} \mathrm{O}_{3} \gamma$ catalyst calculated by the $\mathrm{H}_{2}+\mathrm{O}_{2}$ titration method and electron microscopy [32] $(1 \mathrm{~nm}=10 \AA)$.]

\begin{tabular}{ccc} 
& \multicolumn{2}{c}{ Diamètres (nm) } \\
Microscopie \\
$\% \mathrm{Pt}$ & $\mathrm{H}_{2}+\mathrm{O}_{2}$ & $\begin{array}{c}\text { électronique } \\
-\end{array}$ \\
$\overline{1,0}$ & 1,5 & - \\
2,0 & 1,55 & 1,55 \\
3,0 & 1,5 & 1,54 \\
4,0 & 2,2 & 1,6 \\
3,0 & 8,4 & 1,8 \\
5,0 & 5,7 & 8,7 \\
& & 3,5
\end{tabular}

Les résultats obtenus par mesure de l'hydrogène chimisorbé « irréversiblement » à $200^{\circ} \mathrm{C}$ en choisissant comme stœechiométrie de surface $\mathrm{H} / \mathrm{Pt}_{\mathrm{s}}=1$ et du monoxyde de carbone chimisorbé à $15^{\circ} \mathrm{C}$ avec la stochiométrie $\mathrm{CO} / \mathrm{Pt}_{\mathrm{s}}=1$ sont également en bon accord [32] avec la diffusion centrale des rayons $X$ et la microscopie électronique, comme l'illustre le tableau III.

$$
\begin{aligned}
& \text { Diamètre (nm) } \\
& \text { Rayon X } \\
& \text { (petits angles) }
\end{aligned}
$$

Microscopie électronique

$\begin{array}{ll}\overline{1,3} & \overline{1,6} \\ \overline{2,2} & \overline{1,6} \\ \overline{2,7} & \overline{2,4} \\ 3,2 & \overline{-} \\ 3,0 & 2,3 \\ 4,4 & 3,3 \\ 4,6 & 5,3\end{array}$


Il est à noter que les volumes d'hydrogène adsorbés dans les conditions décrites précédemment ne correspondent pas à la saturation. Nous avons en effet montré que le volume adsorbé à saturation est environ deux fois plus élevé que celui correspondant aux condi-

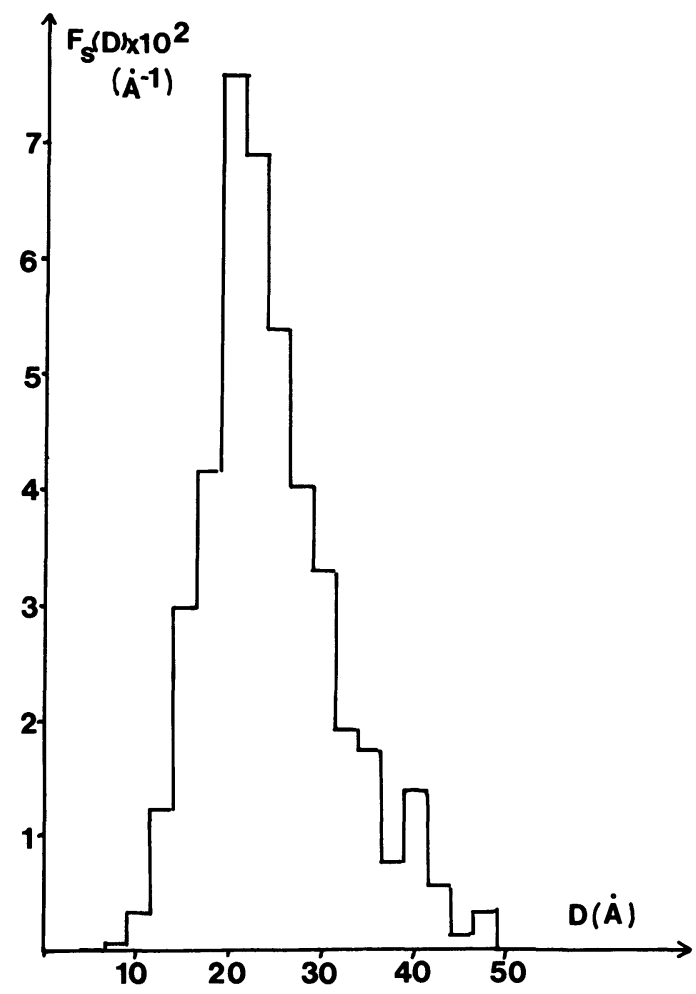

Fig. 5. - Distribution de la taille des grains de platine d'un catalyseur $\mathrm{Pt} / \mathrm{SiO}_{2}$ à $6,3 \%$ de métal. Le diamètre moyen de surface, $D_{\mathrm{s}}=22 \AA(10 \AA=1 \mathrm{~nm})$ est très voisin de celui calculé par diffusion centrale des rayons $X D_{\mathrm{s}}=19 \AA$, et adsorption d'hydrogène $D=20 \AA$ (en admettant qu'à saturation la stœchiométrie de surface est $\mathrm{Pt}_{\mathrm{s}} / \mathrm{H}=1 / 2$ ).

[Distribution of platinum particles size of a $\mathrm{Pt} / \mathrm{SiO}_{2}$ catalyst (6.3 $\mathrm{wt} \%$ in platinum). The mean surface diameter, $D_{\mathrm{s}}=22 \AA$, is nearly equal to that calculated from low angle X-Ray scattering, $D_{\mathrm{s}}=19 \AA$, and hydrogen adsorption $D_{\mathrm{s}}=20 \AA$ (assuming that the surface stoechiometry at saturation is $\left.\mathrm{Pt}_{\mathrm{s}} / \mathrm{H}=1 / 2\right)$.] tions de mesure précédentes, de sorte que la stoechiométrie de surface est à saturation à $\mathrm{H} / \mathrm{Pt}_{\mathrm{s}}=2$ (Fig. 5).

Quelques auteurs estiment [33] que dans certains cas les méthodes faisant appel à la chimisorption surestiment le taux de dispersion du métal par suite d'un effet de migration de $\mathrm{H}_{2}$ sur le support (spill over). C'est pourquoi ils proposent une méthode de mesure de l'aire métallique en faisant réagir un alcène sur le catalyseur saturé en hydrogène (méthode de Bond).

Les réactions d'hydro-déshydrogénation sont en principe caractéristiques de la phase métallique, de sorte que l'on peut espérer atteindre de cette manière l'aire proprement métallique : la réaction est effectuée dans un microréacteur catalytique; après réduction sous $\mathrm{H}_{2}$ puis traitement dans un courant de $\mathrm{N}_{2}$, du 1-pentène est introduit dans le courant gazeux sous pression constante. Les produits de la réaction sont analysés par chromatographie. La teneur en pentane à la sortie du réacteur se maintient à $100 \%$, puis chute brusquement; les auteurs admettent que cette diminution rapide correspond à la consommation d'hydrogène lié au métal; les diamètres des grains du Pt sont calculés d'après la quantité de pentène consommée. Cette méthode qui a été utilisée avec succès pour caractériser les $\mathrm{Pt} / \mathrm{Al}_{2} \mathrm{O}_{3} \gamma$, est toutefois compliquée par la rétention et le craquage de l'hydrocarbure.

4. Conclusion. - Cette revue des techniques généralement utilisées en catalyse pour estimer l'état de dispersion des catalyseurs et la description de quelques systèmes catalytiques usuels indiquent clairement que chacune des méthodes possède ses propres limites et que l'utilisation simultanée de plusieurs techniques est indispensable à une bonne connaissance de la dispersion du catalyseur. La mise en ouvre conjointe de ces méthodes permet actuellement de se faire une idée correcte de la taille des grains de la plupart des masses catalytiques. Les mesures de dispersions, dont la connaissance est indispensable aux études de frittage et à la mesure de l'activité intrinsèque des catalyseurs, contribuent à faire de la catalyse une science quantitative.

\section{Bibliographie}

[1] Boudart, M., Aldag, A., Benson, J. E., Dougharty, N. A. et Harkins, C. G., J. Catal. 6 (1966) 92.

[2] Brunauer, S., Emmet, P. H. et Teller, E., J. Am. Chem. Soc. 60 (1938) 309.

[3] Chon, H., Fisher, R. A., Tomeszko, E. et Aston, J. G., Actes Congr. Int. Catalyse 2, Paris, 1960, vol. 1 (Edit. Technip, Paris) 1961217

[4] Benesi, H. A., Atkins, L. T. et Mosely, R. B., J. Catal. 23 (1971) 211.

[5] Magnan, C., Traité de Microscopie Electronique (Hermann, Paris), 1961.

[6] Eberhart, J. P., Méthodes Physiques des Minéraux et des Matériaux Solides (Doin Editeur) 1976.
[7] Guinier, A., X-Ray: Diffraction (Edit. Freeman and Co San Francisco and London) 1963, et Théorie et Te'(hnique') de la Radiocristallographie (Dunod, Paris) 1956.

[8] SCherRer, P., Nach. Ges. Wiss. Göttingen (1918) 98.

[9] Warren, B. E., J. Appl. Phys. 12 (1941), 375; Anantharaman, T. R., Christian, J. W., Acta crystal 9 (1956) 479.

[10] Porod, G., Kolloid. Z. 124 (1951) 51 ; 125 (1952) 109.

[11] Whyte, T. E., Kirkuin, P. W., Gould, R. W. et HeineMANN, H., J. C'atal. 25 (1972) 407.

[12] de Montgolfier, P., Martin, G. A. et Dalmon, J. A., J. Phys. Chem. Solids 34 (1973) 801.

[13] NeEl, L., Ann. Géophys. 5 (1949) 99.

[14] WeIL, L., J. Chim. Phys. 51 (1954) 775. 
[15] Martin, G. A. et Imelik, B., C. R. Hebd. Séan. Acad. Sci. 270 (1970) 127.

[16] Vannice, M. A., Benson, J. E., Boudart, M., J. Catal. 16 (1970) 348.

[17] Benson, J. E., Boudart, M., J. Catal. 16 (1970) 348.

[18] Candy, J. P., Fouilloux, P. et Renouprez, A. J., J. Chem. Soc. Faraday Trans. 76 (1980) 616.

[19] Fouilloux, P. et Martin, G. A., J. Catal. 38 (1975) 665.

[20] Candy, J. P., Fouilloux, P., Martin, G. A., Blanc, B. et Imelik, B., Fine Particles, W. E. Kuhn, J. Ehretsman Edit. (1974) p. 218.

[21] Prettre, M., C. R. Hebd. Séan., Acad. Sci. 265 série C (1967) 957.

[22] Goue, B., Lenfant, P., C. R. Hebd. Séan. Acad. Sci. 261 série C (1965) 709.

[23] Charcosset, H., Barthomeuf, D., Nicolova, R., Revillon, A., Tournayan, L., Trambouze, Y., Bull. Soc. Chim. 12 (1967) 4555.
[24] Taylor, W. F., Yates, D. J. C. et Sinfelt, J. H., J. Catal. 4 (1965) 274.

[25] Carter, J. L., Cusumano, J. A. et Sinfelt, J. H., J. Phys. Chem. 70 (1966) 2257.

[26] Selwood, P. W., Chemisorption and Magnetization (Academic Press, New York) 1975.

[27] Martin, G. A., J. Catal. 60 (1979) 345.

[28] Martin, G. A. et De Montgolfier, Ph., J. Chim. Phys. 72 (1975) 405.

[29] Martin, G. A., Ceaphalan, N., De Montgolfier, P. et IMELIK, B., J. Chim. Phys. 10 (1973) 1422.

[30] De Montgolfier, Ph., Moraweck, B., Martin, G. A., RenouPRez, A. J. et Dalmai-Imelik, G., Fine Particles, W. E. Kuhn, J. Ehretsman Edit. (1974) p. 43.

[31] Benson, J. E. et Boudart, M., J. Catal. 4 (1965) 704.

[32] Renouprez, A., Hoang Van, C. et Compagnon, P., J. Catal. 34 (1974) 411.

[33] Bond, G. C. et Sermon, P. A., Reaction Kinetics and Catalysis Letters 1 (1974) 3. 